\title{
The Effects of Walking Speed on Adult Multi-segment Foot Kinematics
}

\section{Grant $\mathbf{J}$ and Chester $\mathbf{V}^{*}$}

Andrew and Marjorie McCain Human Performance Laboratory, Faculty of Kinesiology, University of New Brunswick, Fredericton, New Brunswick, Canada E3B 5A3

\begin{abstract}
It has recently been shown that gait speed has an effect on multi-segment foot kinematics. To increase our understanding of these effects, it is critical to compare and develop a database of walking speed ranges. Therefore, the purpose of this study was to determine the effects of walking speed on multi-segment foot kinematics. A 12-camera Vicon motion capture system was used to collect kinematic data on 22 participants (mean age: $23 \pm 2.6$ years). Participants were asked to walk at 5 gait speeds; very slow, slow, free, fast, and very fast. Across speeds, significant differences were found in all absolute angles, 6 of 9 relative mean maximum angles, 11 of 13 timing to the maximum angles, and all temporal-spatial variables analysed. These findings suggest that clinical gait data that uses multi-segment foot models should be matched to a control group walking at a similar speed.
\end{abstract}

Keywords: Multi-segment foot models; Kinematics; Gait; Motion capture

\section{Introduction}

To date, most gait studies have modeled the foot as a single rigid segment with one to two degrees of freedom. Previous research using such models has shown that walking speed influences ankle motion [1-6]. While multi-segment foot models are now more commonly used in gait analysis studies, relatively little is known about the effects of walking speed on subsegment kinematics. Differences in multisegment foot kinematics have been found between control and clinical groups with foot and ankle impairments [7-11]. In many cases, these individuals may walk slower or faster than typical walking speeds. As a result, speed-mediated effects may be difficult to distinguish from those due to an injury, disease and/or disorder. Therefore, a greater appreciation of the effects of walking speed on multi-segment foot kinematics in control groups is needed.

Only a few studies have examined the effects of gait speed on multi-segment foot motion. Dubbeldam et al. [12] measured adult foot and ankle kinematics at self-selected walking speeds and at $75 \%$ and $50 \%$ of self-selected walking speeds. Results showedchanges in multi-segment foot kinematics in all three planes. As speed categories were based on a predetermined percentage of the subjects' self-selected speed, slower speeds may not have reflected each individual's natural movement patterns. Further, while patients usually walk slower than a control group, there are instances where they may walk faster, (e. g., in cases of cerebral palsy). Fast walking speeds were not tested as part of this study. A broader range of speeds would therefore be needed to provide speed-matched data when evaluating a patient. Tulchin et al. [6] studied sagittal plane multi-segment foot kinematics across a range of slow and fast walking speeds in adults using a two-joint model of hind foot and forefoot motion. They found significant changes in hind foot and forefoot motion as a function of speed. However, speed was controlled using a treadmill, which has been shown to impact the natural gait of individuals by altering the frequency and speed relationship [13]. In addition, as the use of treadmills is often impractical in clinical studies, data collected during over ground walking are often preferred for comparative purposes. Deviations in multi-segment foot motion may be related to changes in walking speed that are associated with gait pathology. Kinematic comparisons to speed-matched control data could provide more insight into the effects of pathology on gait. The appreciation of speed-mediated effects on multi-segment foot kinematics is critical for increasing our understanding of foot mechanics in typical and atypical populations. Therefore, the purpose of the present study was to examine differences in multi-segment foot kinematics as a function of walking speed using healthy controls. To facilitate natural gait patterns and applicability for future clinical studies, a broad range of self-selected walking speeds during over-ground walking were used.

\section{Method}

\section{Participants}

Twenty-two adults (11 female, 11 male) aged 18 to 28 years were recruited to participate in the study. One individual was removed from the dataset due to the presence of abnormal gait patterns $(>3$ S.D from control values) for a total of 21 participants(age $=23.0 \pm 2.6$ years; height $=1.73 \pm 0.1 \mathrm{~m}$; weight $=72.1 \pm 10.1 \mathrm{~kg}$ ). One individual's data was removed only from the very slow speed condition due to the onset of toe walking at this speed. Further characteristics of each group are provided in Table 1. Individuals with history of diseases/disorders that could affect foot kinematics, including diabetes, neurological pathologies, joint replacement surgeries, chronic pain, and edema were excluded from the study. Participants were recruited through advertisements, emails, and word-of-mouth. This study was approved by the University Research Ethics Board.

\section{Instrumentation}

Data collection occurred at the Andrew and Marjorie McCain Human Performance Laboratory at the University of New Brunswick (UNB). A12-camera Vicon T160 motion capture system (Oxford Metrics Group Ltd., UK), sampling at $100 \mathrm{~Hz}$, was used to track the three-dimensional trajectories of reflective markers (diameter of $9 \mathrm{~mm}$ ) placed on the participants' skin. Sixforce plates (Kistler Instruments,

*Corresponding author: Chester V, Andrew and Marjorie McCain Human Performance Laboratory, Faculty of Kinesiology, University of New Brunswick Fredericton, New Brunswick, Canada E3B 5A3, Tel: 1-506-458-7589; E-mail: vchester@unb.ca

Received: June 02, 2015; Accepted: June 23, 2015; Published: July 01, 2015

Citation: Grant J, Chester V (2015) The Effects of Walking Speed on Adult Multi-segment Foot Kinematics. J Bioengineer \& Biomedical Sci 5: 156. doi:10.4172/2155- 9538.1000156

Copyright: ( 2015 Grant J, et al. This is an open-access article distributed under the terms of the Creative Commons Attribution License, which permits unrestricted use, distribution, and reproduction in any medium, provided the original author and source are credited. 


\begin{tabular}{|c|c|c|c|c|}
\hline & Number of Participants & Age (years) & Height (m) & Weight (kg) \\
\hline Male & 11 & $22.4(2.7)$ & $1.78(0.06)$ & $76.8(8.5)$ \\
\hline Female & 10 & $23.6(2.5)$ & $1.67(0.05)$ & $67(9.4)$ \\
\hline Combined & 21 & $23(2.6)$ & $1.73(0.1)$ & $72.1(10.1)$ \\
\hline
\end{tabular}

Table 1: Participant characteristics displayed as mean (S.D.).

\begin{tabular}{|l|l|}
\hline Segment & Location \\
\hline Hallux & Most distal and dorsal point of the head of the proximal phalanx \\
\hline Forefoot & Head of the fifth metatarsal, dorso-lateral aspect of the fifth metatarso-phalangeal joint \\
\hline & Head of the second metatarsal, dorso-medial aspect of the second metatarso-phalangeal joint \\
\hline & Head of the first metatarsal, dorso-medial aspect of the first metatarso-phalangeal joint \\
\hline & Base of the fifth metatarsal, dorso-lateral aspect of the fifth metatarso-cuboid joint \\
\hline & Base of the second metatarsal, dorso-medial aspect of the second metatarso-cuneiform joint \\
\hline & Medial apex of the navicular tuberosity \\
\hline Midfoot & Dorsal aspect of the cuboid \\
\hline & Lateral calcaneus-midpoint between the lateral malleolus and floor \\
\hline Calcaneus & Medial calcaneus-point between medial malleolus and floor at height of lateral calcaneus marker \\
\hline & Posterior calcaneus-aligned vertically with Achilles tendon at same height as lateral calcaneus marker \\
\hline & Most lateral aspect of lateral malleolus \\
\hline Shank & Most medial aspect of the medial malleolus \\
\hline & Most lateral prominence of lateral epicondyle \\
\hline & Most medial prominence of the medial epicondyle \\
\hline & Most anterior aspect of the tibial tuberosity \\
\hline
\end{tabular}

Table 2: Anatomical landmarks for the multisegment model of the foot and shank.

Winterthur, Switzerland), embedded in the lab floor,were used to aid in the identification of key gait events. Three-dimensional force and moment data were sampled at a frequency of $1000 \mathrm{~Hz}$. Two custommade laser trip timers were used to provide the researcher with data on walking speeds during the gait trials. Two laser beams were placed $5 \mathrm{~m}$ apart, along the direction of progression, and were integrated with an $\mathrm{A} / \mathrm{D}$ board and laptop computer. Two high-speed Basler digital video cameras (Basler Inc., Ahrensburg, Germany) were used to obtain front and side images of each participant during the gait trials. A weight scale and stadiometer were used to obtain anthropometric measures from each participant.

\section{Procedures}

Thirty four reflective markers were placed on the left and right foot and tibia of each participant (Table 2). For consistency, the same researcher was always responsible for placing markers on each participant in the study. Following this, a static capture of the participant during quiet standing in the anatomical position was recorded to permit the calculation of offset values for all joint rotations. These joint offset values were later subtracted from the appropriate joint rotations for the gait cycles of each participant. These offset calculations did not apply to the planar angles. Following the static trial, several practice gait trials were completed to allow the participants to adjust to the markers and the lab environment. For the gait trials, participants were asked to walk at five different speeds (very slow, slow, free, fast, and very fast). All participants performed free speed trials first to establish a baseline walking speed. Following this, participants were randomly assigned to walk either the slow conditions (e. g. slow, very slow) or the fast conditions (e. g. fast, very fast). Between each of the speed conditions, participants conducted free speed trials, which were not recorded, to aid in re-establishing baseline walking speed. During the gait trials, walking speed data was obtained from the laser trip timers at the completion of each trial. A separation of at least $0.2 \mathrm{~m} / \mathrm{s}$ between speeds was required. Verbal instructions to go faster or slower were given to participants to ensure this separation occurred. Participants were asked to perform at least 6 successful gait cycles (i. e. clean force plate strikes, marker visibility, and appropriate speed separation)at each speed for both the left and right limb $(5$ speeds $\times 2$ limbs $\times 6$ cycles $=60$ cycles per person). Following completion of the gait trials, height and mass were measured.

\section{Data analysis}

Data was analyzed using custom software created in Matlab (Mathworks, Inc. Natick, MA, USA). For each participant, trial selection involved the computation of cadence, velocity, and percent of cycle spent in single stance for each gait cycle. The single gait cycle that most closely approximated the individual mean of all gait cycles on these three measures was selected as the single trial for analysis for each participant. This was repeated for both the left and right limbs.

The rigid body model consisted of five segments: 1) the shank, 2) the total foot (single rigid segment), 3) the calcaneus, 4) the mid foot, and 5) the forefoot (included all 5 metatarsal bones). The hallux and metatarsal bones were modeled as line segments for the computation of planar angles. The anatomical landmarks (Table 2) and reference frames were consistent with the model developed by Leardini et al. [14], with the following exceptions: 1) the cuboid was not assumed to coincide with the base of the fifth metatarsal (Table 2), and 2) a neutral calcaneus was formed using a laser level technique to guide marker placement. This ensured the reliable placement of markers on a segment with few palpable anatomical landmarks. To achieve a neutral calcaneus, the vertical midpoint between the floor and the lateral malleolus was determined using calipers. A marker was placed at this point and referred to as the lateral calcaneus. A cross-hair laser was then used to aid in the placement of the posterior calcaneus marker. The vertical laser line was aligned with midsection of the Achilles tendon, 
Citation: Grant J, Chester V (2015) The Effects of Walking Speed on Adult Multi-segment Foot Kinematics. J Bioengineer \& Biomedical Sci 5: 156. doi:10.4172/2155-9538.1000156

Page 3 of 9

while the horizontal laser line was aligned with the lateral calcaneus marker. The intersection of the two laser lines formed the location for the posterior calcaneus marker. Lastly, the medial calcaneus marker was placed beneath the medial malleolus at the same height as the lateral and posterior calcaneus markers. The locations of the three noncollinear markers on each rigid segment were used to create embedded coordinate systems at the virtual joint centers [14]. Joint angles were computed from the relative orientations of the embedded coordinate systems using Euler angles in ayxz sequence, corresponding to flexion/ extension, adduction/abduction, and internal/external rotation. Displacement data were filtered using a zero phase lag, second order Butterworth filter with a cutoff frequency of 6-9 Hz. Joint angle data were normalized to $100 \%$ of the gait cycle.

In accordance with Hof [15], gait speed was rendered dimensionless by using the following equation:

$$
\widehat{v}=\frac{v}{\sqrt{ } \mathbf{g l}_{0}}
$$

whee $\mathrm{v}$ is the speed, $\mathrm{g}$ is gravity, and $\mathrm{l}_{\mathrm{o}}$ represents the leg length of the individual. The leg length is measured from the greater trochanter to the ground. Speed ranges for each speed condition were determined with respect to these dimensionless values.

\section{Statistical snalysis}

Kinematic data were pooled only after a MANOVA, for the free speed group showed no significant differences $(p<0.05)$ in mean kinematic data between side $(\mathrm{p}=0.133)$ and $\operatorname{sex}(\mathrm{p}=0.366)$. Data for all subjects were then combined and separated into 5 speed groups (very slow, slow, free, fast, and very fast). Repeated measures ANOVAs were used to test for significant $(\mathrm{P}<0.05)$ differences in mean maximum/ minimum joint angles and mean temporal-spatial parameters across the 5 speed groups. A list of dependent variables can be found inTable 3. Bonferroni adjustments were used to compensate for multiple comparisons, which resulted in a p-value of 0.002 or less as significant. All statistical tests were performed using SPSS (IBM Corporation, New York, USA).

\section{Results}

\section{Post HOC analysis of walking speed}

Speed ranges were determined in a post hoc manner by first computing the median walking speed for all trials. A percentage of the median value was then added and subtracted from the median value until approximately one fifth of the data was encompassed by the range of speed values (Table 4). These two initial boundaries defined the free speed range. To define the next speed group (e. g. fast), the percentage

\begin{tabular}{|c|c|c|c|c|c|c|c|c|c|c|}
\hline Kinematics Variables & $\begin{array}{c}\text { Very Slow } \\
\text { Mean }\end{array}$ & SD & Slow & SD & $\begin{array}{l}\text { Free } \\
\text { Mean }\end{array}$ & SD & Fast Mean & SD & $\begin{array}{l}\text { Very fast } \\
\text { mean }\end{array}$ & SD \\
\hline Max v2g (degrees) & 68.5 & 9.08 & 77.73 & 7.42 & 80.25 & 6.98 & 84.82 & 4.85 & 85.6 & 4.65 \\
\hline Time to max v2g (\%GC) & 66.21 & 2.61 & 63.83 & 1.22 & 62.33 & 1.66 & 60.74 & 1.33 & 59.05 & 1.61 \\
\hline Max s2g (degrees) & 93.27 & 8.75 & 101.77 & 8.6 & 105.9 & 7.92 & 109.55 & 6.23 & 111.3 & 6.26 \\
\hline Time to max s2g (\%GC) & 66.16 & 2.63 & 63.93 & 1.27 & 62.45 & 1.76 & 61.23 & 1.39 & 59.82 & 1.47 \\
\hline Max F2Ps (degrees) & 45.05 & 9.28 & 47.35 & 8.4 & 47.75 & 8.16 & 49.55 & 8.5 & 51.86 & 7.1 \\
\hline Time to max F2Ps (\%GC) & 62.95 & 2.52 & 60.1 & 1.64 & 58.69 & 1.7 & 56.84 & 1.79 & 54.56 & 2.21 \\
\hline Min MLA (degrees) & 158.31 & 4.28 & 156.99 & 4.6 & 61.32 & 5.17 & 155.83 & 5.29 & 154.97 & 4.56 \\
\hline Time to $\min$ MLA (\%GC) & 64.91 & 2.28 & 62.95 & 1.56 & 20.65 & 1.55 & 59.74 & 1.35 & 58.05 & 1.61 \\
\hline Max Foot-tibia PF (degrees) & 15.05 & 5.28 & 19.65 & 6.16 & 61.95 & 5.99 & 22.19 & 5.05 & 22.73 & 5.41 \\
\hline Time to max Foot-Tibia PF (\%GC) & 53.44 & 25.1 & 60.76 & 12.9 & 9.98 & 1.67 & 60.53 & 1.5 & 58.59 & 1.79 \\
\hline Max Foot-tibia DF (degrees) & 11.02 & 3.1 & 11.21 & 3.02 & 43.31 & 3.5 & 9.04 & 2.79 & 7.77 & 2.98 \\
\hline Time to max Foot-Tibia DF (\%GC) & 49.37 & 3.75 & 46.56 & 2.72 & 15.72 & 6.96 & 40.49 & 7.84 & 32.46 & 9.52 \\
\hline Max Calcaneus-Tibia PF (\%GC) & 11.38 & 3.69 & 14.7 & 4.88 & 54.05 & 5.03 & 17.01 & 4.16 & 17.17 & 3.75 \\
\hline Time to max Calcaneus-Tibia PF (\%GC) & 40.77 & 30.5 & 53.88 & 22.2 & 7.49 & 20.19 & 59.4 & 8.85 & 56.13 & 12.16 \\
\hline Max Calcaneus-Tibia DF (\%GC) & 8.74 & 2.88 & 8.63 & 2.82 & 43.05 & 3.26 & 6.62 & 2.83 & 6.08 & 2.68 \\
\hline Time to max Calcaneus-Tibia DF (\%GC) & 49.47 & 4.05 & 46.73 & 4.15 & 2.06 & 7.88 & 39.91 & 8.44 & 32.15 & 9.64 \\
\hline Max Mid foot-Calcaneus EVE (degrees) & 1.58 & 1.08 & 1.76 & 0.99 & 2.06 & 1.19 & 1.96 & 1.34 & 2.1 & 1.39 \\
\hline Time to Max Midfoot-Calcaneus EVE (\%GC) & 58.23 & 9.12 & 54.34 & 10.5 & 55.88 & 7.3 & 52.33 & 10.27 & 49.56 & 13.59 \\
\hline Max Mid foot-Calcaneus PF (degrees) & 8.8 & 2.34 & 9.85 & 3.2 & 10.09 & 3.17 & 10.1 & 3.25 & 10.52 & 3.92 \\
\hline Time to Max Midfoot-Calcaneus PF (\%GC) & 65.19 & 2.38 & 62.85 & 1.41 & 61.36 & 1.79 & 59.81 & 1.4 & 58.13 & 1.89 \\
\hline $\begin{array}{l}\text { Max Fore Foot-Midfoot Calcaneus EVE } \\
\text { (degrees) }\end{array}$ & 2.5 & 1.57 & 2.73 & 1.74 & 2.9 & 1.91 & 2.79 & 1.88 & 2.99 & 1.77 \\
\hline $\begin{array}{l}\text { Time to Max Fore Foot-Midfoot Calcaneus } \\
\text { EVE (\%GC) }\end{array}$ & 47.95 & 19 & 50.76 & 17 & 50.05 & 17.36 & 51.26 & 14.21 & 54.08 & 12.26 \\
\hline Max Fore Foot-Midfoot CalcaneusPF (degrees) & 10.83 & 2.84 & 12.29 & 3.24 & 12.5 & 3.37 & 12.7 & 3.44 & 13.6 & 3.9 \\
\hline $\begin{array}{l}\text { Time to Max Fore Foot-Midfoot Calcaneus PF } \\
\qquad(\% G C)\end{array}$ & 64.07 & 2.3 & 61.61 & 1.55 & 60.36 & 1.71 & 58.65 & 1.46 & 57 & 1.85 \\
\hline Max Fore foot-calcaneus ADD (\%GC) & 4.79 & 2.46 & 5.67 & 2.85 & 5.09 & 2.9 & 4.84 & 2.99 & 4.2 & 3.13 \\
\hline $\begin{array}{l}\text { Time to Max Fore Foot-Midfoot Calcaneus } \\
\text { ADD (\%GC) }\end{array}$ & 50.14 & 21.8 & 54.34 & 12.1 & 48.86 & 17.66 & 49.93 & 12.35 & 37.23 & 22.56 \\
\hline Stride Length $(\mathrm{cm})$ & 103.68 & 10.3 & 123.02 & 7.58 & 136.5 & 8.65 & 151.24 & 9.97 & 166.85 & 11.48 \\
\hline Toe-Off (\%GC) & 66.33 & 2.88 & 63.95 & 1.26 & 62.45 & 1.76 & 61.26 & 1.42 & 59.87 & 1.47 \\
\hline Single leg Stance (\%GC) & 35.02 & 3.23 & 36.8 & 1.61 & 38.31 & 1.6 & 39.67 & 1.46 & 41.09 & 1.7 \\
\hline Cadence & 106.10 & 14.3 & 125.1 & 8.32 & 140 & 8.09 & 153.18 & 8 & 171.23 & 12.73 \\
\hline
\end{tabular}

Table 3: Descriptive statistics for joint angle and temporal spatial data as a function of walking speed. 
Citation: Grant J, Chester V (2015) The Effects of Walking Speed on Adult Multi-segment Foot Kinematics. J Bioengineer \& Biomedical Sci 5: 156. doi:10.4172/2155-9538.1000156

Page 4 of 9

\begin{tabular}{|c|c|c|}
\hline Speed Groups & Dimensionless Speed & Number of Trials \\
\hline Very Slow & $v \leq 0.382$ & 43 \\
\hline Slow & $0.382<v \leq 0.495$ & 41 \\
\hline Free & $0.495<v \leq 0.581$ & 42 \\
\hline Fast & $0.58<v \leq 0.721$ & 43 \\
\hline Very Fast & $v>0.721$ & 39 \\
\hline
\end{tabular}

Table 4: Post hoc classification of walking speeds among the five speed categories.

\begin{tabular}{|c|c|c|c|c|c|}
\hline Dependent Variable & Very Slow & Slow & Free & Fast & Very Fast \\
\hline MaximumV2G (fifth metatarsal in the sagittal plane) & $2,3,4,5$ & $1,4,5$ & $1,4,5$ & $1,2,3$ & $1,2,3$ \\
\hline Time to maximumV2G & $2,3,4,5$ & $1,3,4,5$ & $1,2,4,5$ & $1,2,3,5$ & $1,2,3,4$ \\
\hline MaximumS2G (second metatarsal in the sagittal plane) & $2,3,4,5$ & $1,3,4,5$ & $1,2,4,5$ & $1,2,3$ & $1,2,3$ \\
\hline Time to maximumS2G & $2,3,4,5$ & $1,3,4,5$ & $1,2,4,5$ & $1,2,3,5$ & $1,2,3,4$ \\
\hline Maximum F2Ps(hallux in the sagittal plane) & $2,3,4,5$ & $1,4,5$ & 1,5 & 1,2 & $1,2,3$ \\
\hline Time to maximum F2Ps & $2,3,4,5$ & $1,3,4,5$ & $1,2,4,5$ & $1,2,3,5$ & $1,2,3,4$ \\
\hline Minimum MLA(medial longitudinal arch) & $2,3,4,5$ & 1,5 & 1 & 1 & 1,2 \\
\hline Time to minimum MLA & $2,3,4,5$ & $1,3,4,5$ & $1,2,4,5$ & $1,2,3,5$ & $1,2,3,4$ \\
\hline Maximum Foot-Tibia PF & $2,3,4,5$ & $1,4,5$ & 1,5 & 1,2 & $1,2,3$ \\
\hline Time to maximum Foot-Tibia PF & $2,3,4,5$ & 1 & 1 & 1 & 1 \\
\hline Maximum Foot-Tibia DF & 4,5 & 4,5 & 5 & $1,2,5$ & $1,2,3,4$ \\
\hline Time to maximum Foot-Tibia DF & $3,4,5$ & $3,4,5$ & $1,2,5$ & $1,2,5$ & $1,2,3,4$ \\
\hline Maximum Calcaneus-Tibia PF & $2,3,4,5$ & 1,5 & 1 & 1 & 1,2 \\
\hline Time to maximum Calcaneus-Tibia PF & $2,3,4,5$ & 1 & 1 & 1 & 1 \\
\hline Maximum Calcaneus-Tibia DF & $3,4,5$ & 4,5 & 1,5 & 1,2 & $1,2,3$ \\
\hline Time to maximum Calcaneus-Tibia DF & $3,4,5$ & $3,4,5$ & $1,2,5$ & $1,2,5$ & $1,2,3,4$ \\
\hline Maximum Midfoot-Calcaneus PF & $2,4,5$ & 1 & 5 & 1 & 1,3 \\
\hline Time to maximum Midfoot-Calcaneus PF & $2,3,4,5$ & $1,3,4,5$ & $1,2,4,5$ & $1,2,3,5$ & $1,2,3,4$ \\
\hline Maximum Forefoot-Calcaneus PF & $2,3,4,5$ & 1,5 & 1,5 & 1 & $1,2,3$ \\
\hline Time to maximum Forefoot-Calcaneus PF & $2,3,4,5$ & $1,3,4,5$ & $1,2,4,5$ & $1,2,3,5$ & $1,2,3,4$ \\
\hline Time to maximum Forefoot-Calcaneus ADD & 5 & 5 & 5 & 5 & $1,2,3,4$ \\
\hline Stride length & $2,3,4,5$ & $1,3,4,5$ & $1,2,4,5$ & $1,2,3,5$ & $1,2,3,4$ \\
\hline Toe-off & $2,3,4,5$ & $1,3,4,5$ & $1,2,4,5$ & $1,2,3,5$ & $1,2,3,4$ \\
\hline Single leg stance & $2,3,4,5$ & $1,3,4,5$ & $1,2,4,5$ & $1,2,3,5$ & $1,2,3,4$ \\
\hline Cadence & $2,3,4,5$ & $1,3,4,5$ & $1,2,4,5$ & $1,2,3,5$ & $1,2,3,4$ \\
\hline
\end{tabular}

Table 5: Significant pairwise comparisons of temporal-spatial and joint angle data between speed conditions: 1-very slow, 2-slow, 3-free, 4-fast, 5- very fast ( $\mathrm{p}<0.002)$

was increased until an additional one fifth of the data was encompassed. This process was repeated for the remaining speed groups. The post HOC analysis of walking speeds allowed for the formation of relatively equal groupings of trials across speed conditions (Table 4).

\section{Temporal-spatial Parameters}

Significant differences $(\mathrm{p}<0.002)$ were found in the mean values of temporal-spatial variables across all speed conditions (Tables 3 and 5). As gait speed increased, mean stride length increased from $103.68 \mathrm{~cm}$ (very slow) to $166.80 \mathrm{~cm}$ (very fast), the mean percentage of the gait cycle at which toe off occurred decreased from $66.33 \%$ (very slow) to 59 9\% (very fast), and mean single leg stance phase duration increased from $35.02 \%$ (very slow) to $41.1 \%$ (very fast) of the gait cycle. In addition, cadence increased from 106.10 steps per minute (very slow) to 171.23 steps per minute (very fast).

\section{Joint angles}

Descriptive statistics for joint angle measurements as a function of speed are provided in Table 3. Significant pairwise comparisons between speed conditions are listed in Table 5. Significant differences $(p<0.002)$ in the mean maximum dorsiflexion and plantar flexion angles were found between speed conditions for the foot with respect to the tibia and calcaneus with respect to the tibia (Tables 3-5 and Figure
1). As walking speed increased, the mean maximum dorsiflexion angle (foot with respect to tibia) decreased from $11.02^{\circ}$ (very slow) to $7.77^{\circ}$ (very fast), while the maximum plantar flexion angle increased from 15. $05^{\circ}$ (very slow) to $22.73^{\circ}$ (very fast). The calcaneus, with respect to the tibia, exhibited similar results (Figure 1) with a significant decrease in the mean dorsiflexion angle from $8.74^{\circ}$ (very slow) to $6.08^{\circ}$ (very fast), and a significant increase in mean the maximum plantarflexion angle from $11.38^{\circ}$ (very slow) to $17.17^{\circ}$ (very fast). Significant differences in mean maximum joint angles were also found between speeds for the mid foot relative to the calcaneus and the forefoot relative to the calcaneus (Figure 1). As walking speed increased, the mean maximum mid foot-calcaneus plantar flexion angle increased from $8.80^{\circ}$ (very slow) to $10.52^{\circ}$ (very fast). The forefoot with respect to the calcaneus demonstrated similar results with an increase in the mean maximum plantarflexion angle from $10.83^{\circ}$ (very slow) to $13.60^{\circ}$ (very fast). Mean relative angles as a function of gait speed are provided in Figures 2 and 3. In the sagittal plane, significant differences $(p<0.002)$ in the mean maximum absolute angles of the metatarsals and the hallux were found between gait speeds(Figure 4 and Tables 3 and 5). The mean maximum absolute angle for the fifth metatarsal (V2G) increased from $68.50^{\circ}$ (very slow) to $86.60^{\circ}$ (very fast), while the mean maximum angle for the second metatarsal (S2G) increased from $93.27^{\circ}$ (very slow) to $111.30^{\circ}$ (very fast). 
Citation: Grant J, Chester V (2015) The Effects of Walking Speed on Adult Multi-segment Foot Kinematics. J Bioengineer \& Biomedical Sci 5: 156. doi:10.4172/2155-9538.1000156

Page 5 of 9

The maximum absolute sagittal plane angle for the hallux (F2Ps) increased from $45.05^{\circ}$ (very slow) to $51.86^{\circ}$ (very fast) as gait speed increased. The medial longitudinal arch (MLA) showed significant changes in mean maximum angle as a function of speed, decreasing from $158.31^{\circ}$ (very slow) to $154.97^{\circ}$ (very fast). Figure 5 shows mean absolute joint angles across speed conditions. Mean time to maximum angles, as a percentage of gait cycle, were significantly different $(\mathrm{p}<0.002)$ for all measures except time to maximum mid foot-calcaneus eversion, and time to maximum forefoot-mid foot eversion (Tables 3 and 5). Across the 5 gait speeds, there was a decrease in the timing to maximum joint angle values as gait speed increased.

\section{Discussion}

There have been numerous studies on the effects of walking
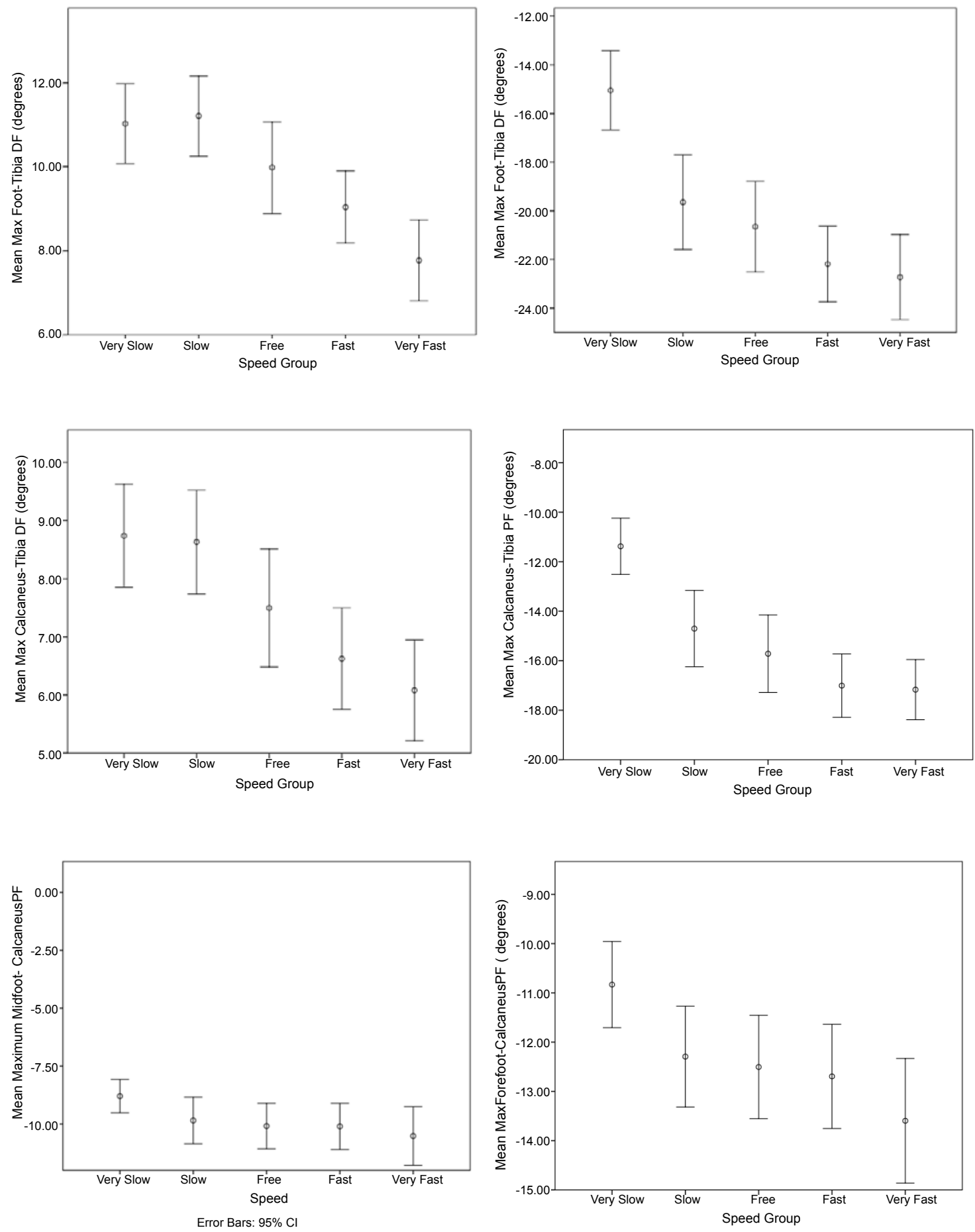

Figure 1: Mean maximum relative angles with $95 \%$ confidence interval for 5 gait speeds. 
speed on lower extremity kinematics and kinetics [1-2,4,6,16-18]. Despite increases in the number of studies using multi-segment foot models, few studies have examined changes in multi-segment foot kinematics as a function of walking speed. Such data could be useful when comparing kinematic results between studies involving differing walking speeds and for clinical gait studies. Therefore, the purpose of this study was to determine the effects of walking speed on multisegment foot kinematics in healthy adults. No significant differences $(\mathrm{p}<0.05)$ in multi-segment foot kinematics were found as a function of gender or limbs (left/right), therefore, the kinematic data was pooled

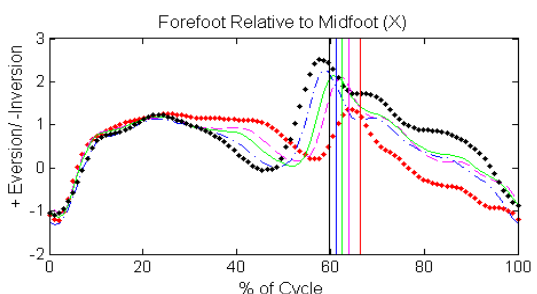

Forefoot relative to Calcaneus $(X)$

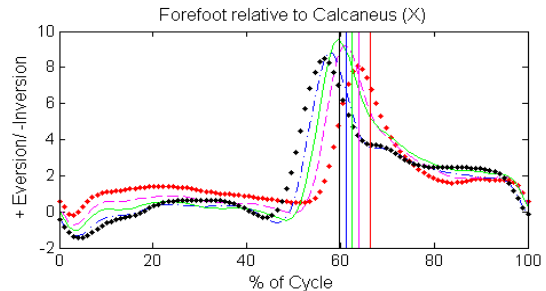

Midfoot Relative to Calcaneus $(X)$

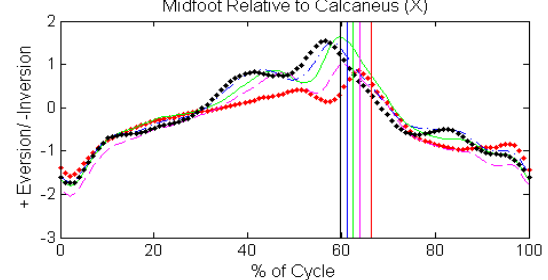

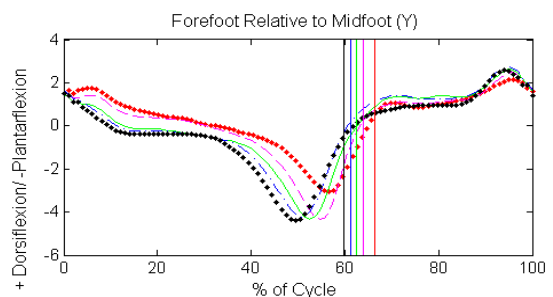

Forefoot relative to Calcaneus $(Y)$

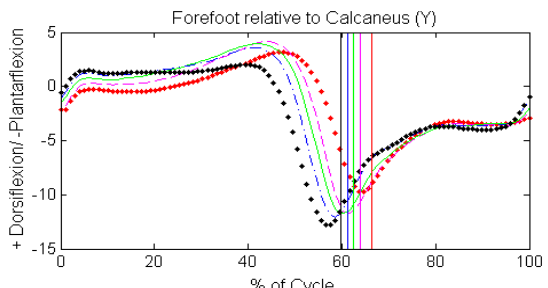

Midfoot Relative to Calcaneus ( $($ )

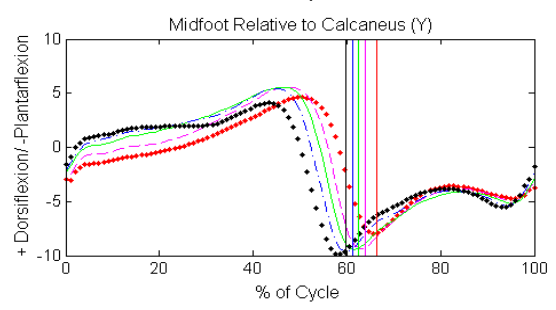

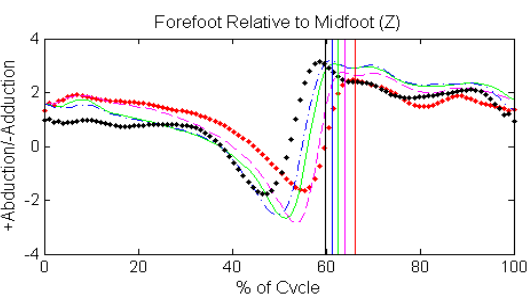

Forefoot relative to Calcaneus (Z)

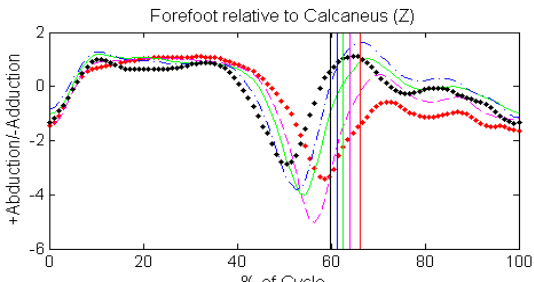

Midfoot Relative to Calcaneus (Z)

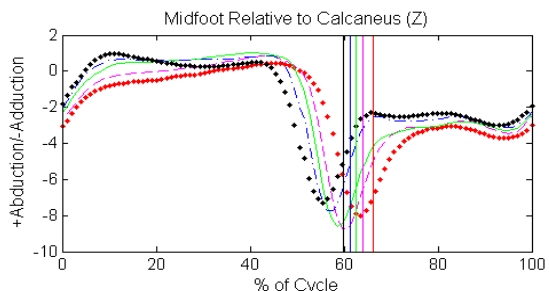

Very Slow

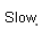

Free

Fast

Very Fast

Figure 2: Mean relative angles during the gait cycle for all 5 speeds.

for all subsequent analyses. Data was collected across a wide range of walking speeds, from $0.6 \mathrm{~m} / \mathrm{s}$ to $2.9 \mathrm{~m} / \mathrm{s}$.

\section{Temporal-spatial data}

As expected, all of the temporal-spatial variables showed significant differences with changes in walking speed (Tables 3 and 5) $(\mathrm{p}<0.002)$. As gait speed increased, time to toe-off decreased from $66.33 \%$ to $59.90 \%$, single leg stance time increased from $35.02 \%$ to $41.1 \%$ of the gait cycle, stride length increased from $103.6 \mathrm{~cm}$ to $166.8 \mathrm{~cm}$ and cadence increased from 106.10 to $171.23 \mathrm{steps} / \mathrm{min}$. Similar results were found by previous researchers [5-6,12].

\section{Joint Angle Data}

Variation in walking speed significantly affected the magnitude and timing of the relative and absolute angles of the foot. All significant changes in the magnitude of angles occurred in the sagittal plane only. The relative angles between the calcaneus and tibia showed significant $(\mathrm{p}<0.002)$ changes in both dorsiflexion and plantar flexion as a function of gait speed (Tables 3 and 5 and Figures 1 and 3). As speed increased, the mean maximum dorsiflexion angle decreased from $8.74^{\circ}$ at very slow to $6.08^{\circ}$ at very fast and the mean maximum plantar flexion angle increased from $11.38^{\circ}$ at very slow to $17.17^{\circ}$ at very fast. Similarly, Dubbeldam et al. [12] analyzed the talus with respect to the tibia and found that plantar flexion angles increased at toe-off as gait speed increased. In the present study, a plateau effect was observed between the very slow and slow speed groups for the mean maximum dorsiflexion angle and between the very fast and fast speed groups for the mean maximum plantar flexion angle. Similar results were found by Schwartz et al. [6] for mean ankle dorsiflexion at toe-off between the slow and slow speeds. It is possible that the point at which the angle plateau occurs may represent a structural limitation in the joint or the magnitude of separation between speeds is not sufficient to alter joint motion. Small but significant changes in mean maximum plantar flexion of the mid foot relative to the calcaneus was found across walking speeds (Tables 3 and 5 and Figures 1 and 2). The maximum plantar flexion of the mid foot-calcaneus changed from $8.8^{\circ}$ at very slow to $10.52^{\circ}$ at very fast. Dubbeldam et al. [12] also included the mid foot segment in their study but did not find significant differences in sagittal plane motion with changes in walking speed. However, they did 

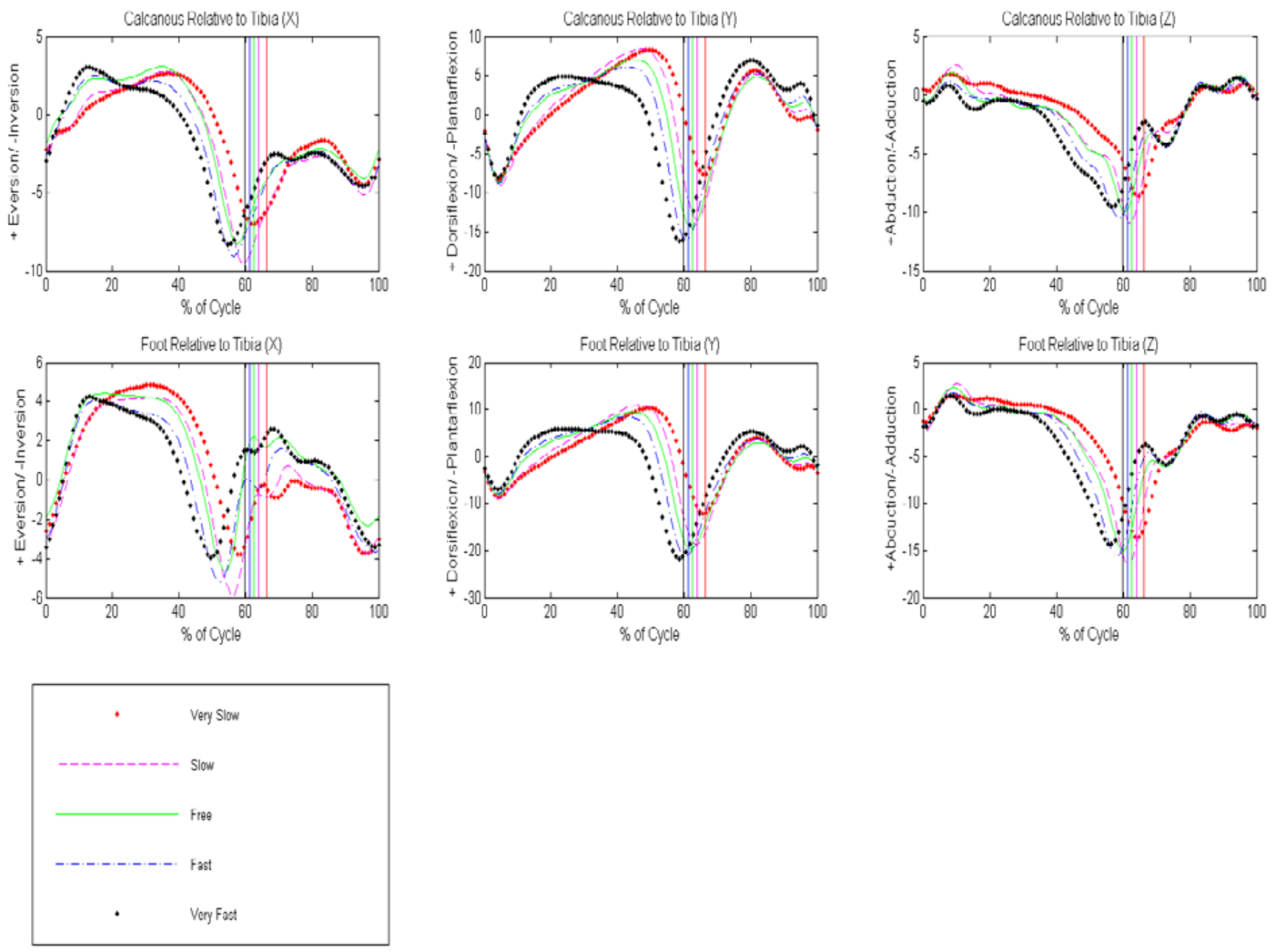

Figure 3: Mean relative angles during the gait cycle for all 5 speeds.

find that pronation of the mid foot relative to the calcaneus increased during midstance with a reduction in gait speed. There were significant differences in the mean maximum plantar flexion angles of the forefoot with respect to the calcaneus across speeds (Tables 3 and 5 and Figures 1 and 2). The mean maximum plantar flexion angle changed from $10.83^{\circ}$ at very slow to $13.6^{\circ}$ at very fast walking speeds. Only small changes in the mean maximum angle were observed between the slow, free, and fast walking speed conditions. Tulchin et al. [5] reporteda mean maximum forefoot-calcaneus plantar flexion angle of approximately $12^{\circ}$ at very slow to approximately $17^{\circ}$ at the very fast walking speed. Differences in walking speeds and the anatomical landmarks used to define the forefoot segment between studies may have contributed to the differential results.

The relative angles of the foot with respect to the tibia (foot as a single segment) showed similar results to that of the calcaneus relative to the tibia (Tables 3 and 5 and Figures 1 and 3). As gait speed increased, mean maximum dorsiflexion angle in the present study decreased from $11.02^{\circ}$ at very slow to $7.77^{\circ}$ at very fast. Similar results were found by Schwartz et al. [6] and Tulchin et al. [5]. In addition, as gait speed increased, there was an increase in the mean maximum plantar flexion angle from $15.05^{\circ}$ at very slow to $22.73^{\circ}$ at very fast. This range is similar to that reported by Tulchin et al. [5], where the mean plantar flexion angle was approximately $15^{\circ}$ at the very slow speed and approximately $25^{\circ}$ at the very fast speed. Schwartz et al. [6] reported a mean plantar flexion angle of approximately $4^{\circ}$, at a very slow speed, and approximately $22^{\circ}$ at a very fast speed. Differences in results at the slower speeds may be due to the varying walking speeds between studies.

There were significant changes in the mean maximum absolute angles as a function of gait speed (Tables 3 and 5 and Figures 4 and 5). The maximum $\mathrm{S} 2 \mathrm{G}$ angle increased from $93.27^{\circ}$ at very slow to $111.3^{\circ}$ at very fast, while the maximum V2G angle increased from $68.5^{\circ}$ at very slow to $86.6^{\circ}$ at very fast. This increase in S2G and V2G indicated that the proximal end of metatarsals 2 and 5, were rotating towards the distal end of the foot as the foot progressed forward towards toeoff. The mean maximum value of F2Ps, dorsiflexion of the hallux, also increased from $45.05^{\circ}$ at very slow to $51.86^{\circ}$ at very fast as gait speed increased. As gait speed increased the mean minimum MLA angle 

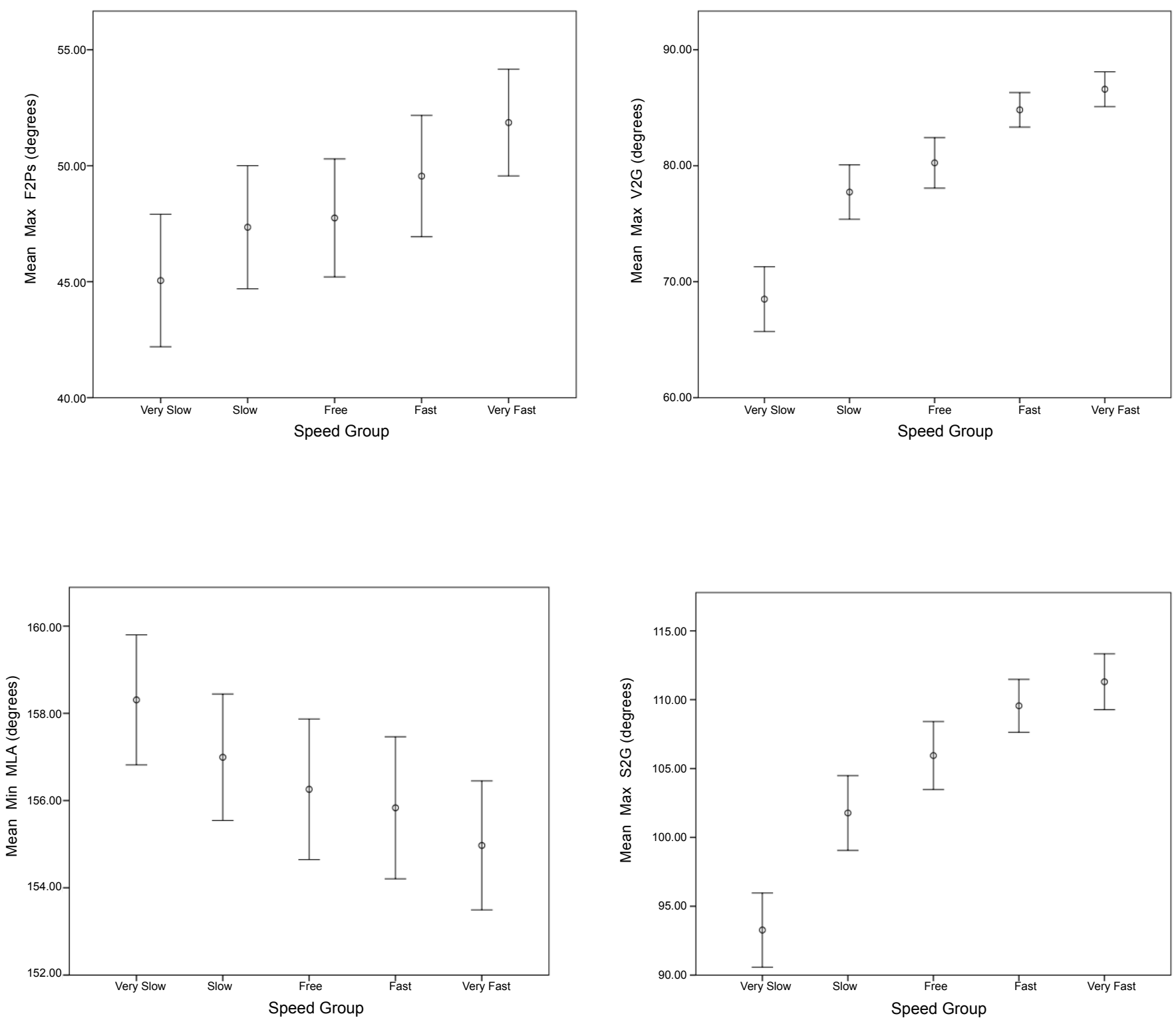

Figure 4: Mean maximum absolute angles with $95 \%$ confidence interval for 5 gait speeds.

changed from $158.31^{\circ}$ at very slow to $154.97^{\circ}$ at very fast. Similar results were reported by Dubbeldam et al. [12] for hallux dorsiflexion and the medial longitudinal arch angle.

Mean time to maximum angles, as a percentage of gait cycle, were significantly different $(\mathrm{p}<0.002)$ for all measures except time to maximum mid foot-calcaneus eversion, and time to maximum forefoot-mid foot eversion (Tables 3 and 5). As expected, mean time to maximum angles decreased as gait speed increased reflecting decreased stance phase duration. The lack of difference in timing for eversion is likely associated with the very small amount of motion between the foot segments in this direction of rotation. Tulchin et al. [5] also found that the timing to maximum hind foot dorsiflexion with respect to the tibia occurred earlier in the gait cycle as gait speed increased.

There are several limitations that must be acknowledged. First, ranges of walking speed may be achieved through numerous mechanisms. In the present study, verbal instructions (e. g. "walk fast" or "walk faster") were used to promote changes in walking speed for each subject. Compared to using treadmills or metronomes, it was hoped that this method would facilitate more natural gait patterns at each speed. The post hoc determination of speed groups (e. g. very fast vs. fast) also led to some unbalanced data. That is, some subjects had multiple trials (e. g. their slow and very slow trials) in the same speed group. This suggests that a greater separation may be needed between speeds. Finally, the walking speeds observed in the present study may not reflect those associated with certain gait impairments. Specifically, slower speeds may need to be examined in order to fully understand the impact on multi segment foot kinematics.

Few studies have examined the effects of walking speed on multi segment foot kinematics. Similar to the single rigid body model, walking speed was found to affect multi segment foot kinematics. 

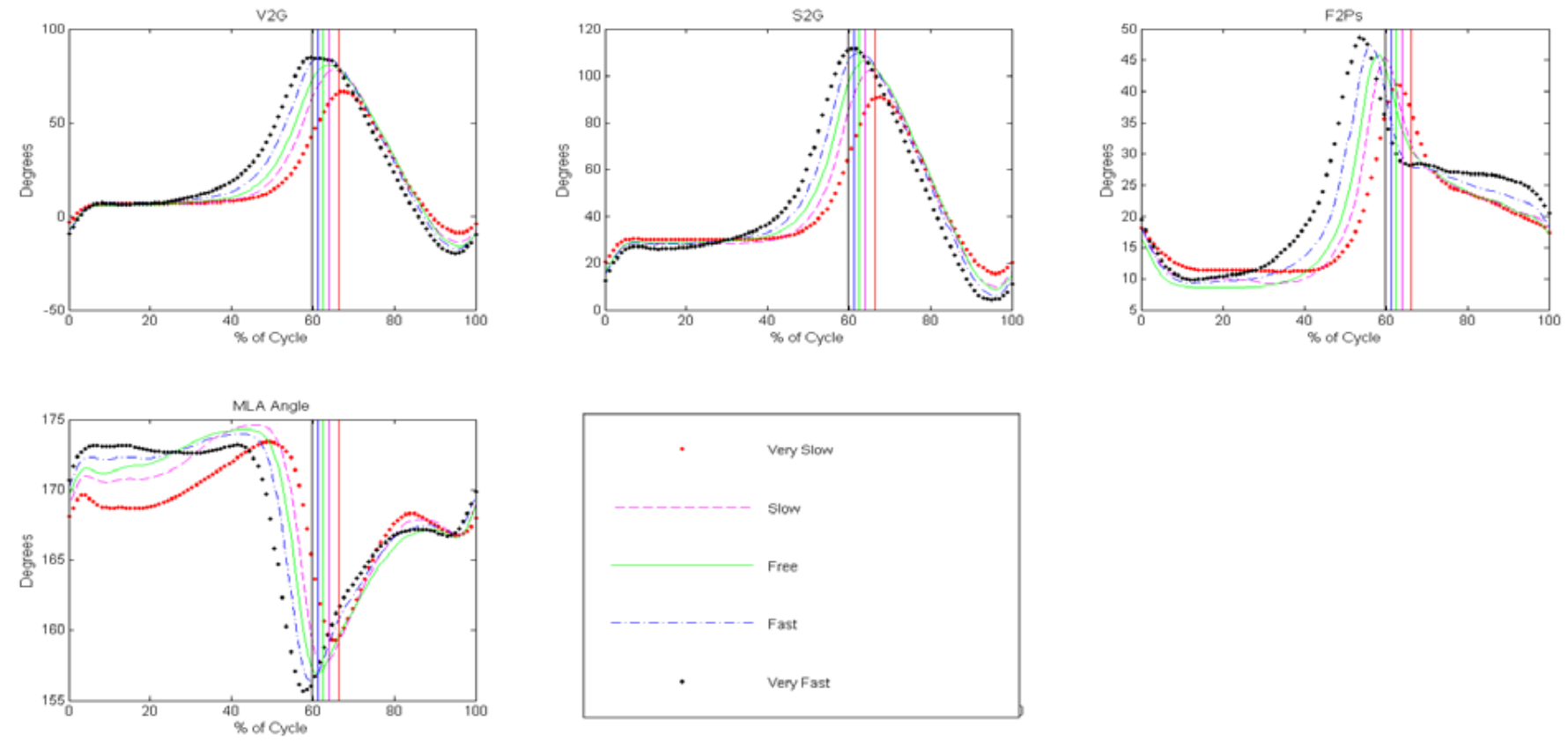

Figure 5: Mean absolute angles during the gait cycle for all 5 speeds.

These results have important implications for studies examining foot and ankle function in typical and atypical populations. The results suggest that speed-matched control data should be used for the analysis of multisegment kinematics, especially in clinical populations where walking speed is altered by pathology. Such data will also facilitate a greater understanding of gait deviations and the effects of treatment interventions. Future studies should examine the effects of walking speed on multisegment foot kinematics in varying age groups and clinical populations. In addition, changes in multisegment foot kinematics as a function of speed may be further understood through the examination of kinetic, EMG, and plantar pressure data.

\section{References}

1. Crowninshield RD, Johnston RC, Andrews JG, Brand RA (1978) A biomechanical investigation of the human hip. J Biomech 11: 75-85.

2. Kirtley C, Whittle MW, Jefferson RJ (1985) Influence of walking speed on gait parameters. JBiomed Eng 7: 282-288.

3. Van der Linden ML, Kerr AM, Hazlewood ME, Hillman SJ, Robb JE (2002) Kinematic and Kinetic Gait Characteristics of Normal Children Walking at a Range of Clinically Relevant Speeds. J Pediatr Orthoped 22: 800-806.

4. Lelas JL, Merriman GJ, Riley PO, Kerrigan DC (2003) Predicting peak kinematic and kinetic parameters from gait speed. GaitPosture 17: 106-112.

5. Tulchin K, Orendurff M, Adolfsen, S, Karol L (2009) The effects of walking speed on multisegment foot kinematics in adults. J Appl Biomech 25: 377-386.

6. Schwartz MH, Rozumalski A, Trost JP (2008) The effect of walking speed on the gait of typically developing children. J Biomech 41: 1639-1650.

7. Woodburn J, Nelson KM, Siegel KL, Kepple T, Gerber LH (2004) Multisegment foot motion during gait: proof of concept in rheumatoid arthritis. Journal Rheumatol 31:1918-1927.

8. Rao S, Saltzman C, Yack HJ (2007) Segmental foot mobility in individuals with and without diabetes and neuropathy. Clin Biomech 22: 464-471.

9. Ness ME, Long J, Marks R, Harris G (2008) Foot and ankle kinematics in patients with posterior tibial tendon dysfunction. Gait Posture 27: 331-339.
10. Alonso-Vazquez A, Villarroya MA, Franco MA, Asin J, Calvo B (2009) Kinematic assessment of paediatric forefoot varus. Gait Posture 29: 214-219.

11. Canseco K, Long J, Marks R, Khazzam M, Harris G (2009) Quantitative Motion Analysis in Patients with Hallux RigidusBefore and After Cheilectomy. J Orthop Research 27: 128-134.

12. Dubbeldam R, Buurke JH, Simons C, Groothuis-Oudshoorn CG, Baan H, et al. (2010) The Effects of Walking Speed on Forefoot, Hindfoot and Ankle Joint Motion. Clin SBiomech 25: 796-801.

13. Bertram JE, Ruina A (2001) Multiple Walking Speed-frequency Relations are Predicted by Constrained Optimization. J Theor Biol 209: 445-453.

14. Leardini A, Benedetti MG, Berti L, Bettinelli D, Nativo R, et al. (2007) Rear-foot Mid-foot and Fore-foot Motion During the Stance Phase of Gait. Gait Posture 25: 453-462.

15. Hof AL (1996) Scaling Gait Data to Body Size. GaitPosture 4: 222-223.

16. Murray MP, Kory RC, Clarkson BH, Sepic SB (1966) Comparison of Free and Fast Speed Walking Patterns of Normal Men. Amer J Physical Med 45: 8-23.

17. Stansfield BW, Hillman SJ, Hazlewood ME, Robb JE (2006) Regression Analysis of Gait Parameters with Speed in Normal Children Walking at Selfselected Speeds. Gait Posture 23: 288-294.

18. Stoquart G, Detrembleur C, Lejeune T (2008) Effect of Speed on Kinematic Kinetic, and Electromyographic and Energetic Reference Values during Treadmill Walking. Journal of Clinical Neurophysiology 38: 105-116. 